\title{
Characterization of Drug-Resistant Salmonella enterica Serotype Typhimurium by Antibiograms, Plasmids, Integrons, Resistance Genes, and PFGE
}

\author{
Benacer, Douadi ${ }^{1}$, Kwai Lin Thong ${ }^{*}$, Haruo Watanabe ${ }^{2}$, and Savithri Devi Puthucheary ${ }^{3}$ \\ ${ }^{1}$ Institute of Biological Sciences, Faculty of Science, University of Malaya, Kuala Lumpur 50603, Malaysia \\ ${ }^{2}$ National Institute of Infectious Disease, Tokyo 162-8640, Japan \\ ${ }^{3}$ Department of Medical Microbiology, Faculty of Medicine, University of Malaya, Kuala Lumpur 50603, Malaysia
}

Received: October 21, 2009 / Revised: March 3, 2010 / Accepted: March 18, 2010

Forty-seven Salmonella Typhimurium (33 zoonotic, 14 clinical) strains were tested for antimicrobial resistance using the standard disk diffusion method. The presence of relevant resistance genes and class 1 integrons were investigated by using PCR. Pulsed-field gel electrophoresis (PFGE) and plasmid profiling were carried out to determine the genomic diversity of Salmonella Typhimurium. Approximately $57.4 \%$ of the $S$. Typhimurium strains were multidrug resistant (MDR) and showed high resistance rates to tetracycline $(\mathbf{7 0 . 2 \%})$, sulfonamides $(\mathbf{5 7 . 4 \%})$, streptomycin $(53.1 \%)$, ampicillin $(29.7 \%)$, nalidixic acid $(27.6 \%)$, kanamycin (23.4\%), chloramphenicol $(21.2 \%)$, and trimethoprim $(19.1 \%)$. Resistance towards cephalosporins was noted for cephalothin $(27.6 \%)$, cephradine $(21.2 \%)$, amoxicillin clavulanic acid (17.0\%), and cephalexin (17.0\%). Resistance

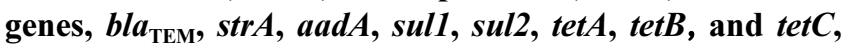
were detected among the drug-resistant strains. Thirtythree strains $(\mathbf{7 0 . 2 \%})$ carried class 1 integrons, which were grouped in 9 different profiles. DNA sequencing identified sat, aadA, pse-1, and $\operatorname{dfr} A$ genes in variable regions on class 1 integrons. Thirty-five strains $\mathbf{( 7 4 . 4 \% )}$ were subtyped to 22 different plasmid profiles, each with 1-6 plasmids (2.0 to $95 \mathrm{~kb}$ ). PFGE subtyped the 47 strains into 39 profiles. In conclusion, high rates of multidrug resistance were found among the Malaysian Salmonella Typhimurium strains. The emergence of multidrug-resistant Salmonella Typhimurium to cephalosporin antibiotics was also observed. The strains were very diverse and no persistent clone was observed. The emergence of MDR Salmonella Typhimurium is a worldwide problem, and this report provides information for the better understanding of the prevalence and epidemiology of MDR $S$. Typhimurium in Malaysia.

* Corresponding author

Phone: +603-79674437; Fax:+603-79675908;

E-mail: thongkl@um.edu.my
Keywords: Antimicrobial resistance, integrons, plasmids, PFGE, $S$. Typhimurium

Human salmonellosis continues to be a major public health problem in both developed and developing countries. Nontyphoidal salmonellosis (NTS), caused by salmonellae other than Salmonella Typhi, is by far the most common cause of bacteremia associated with gastroenteritis. Between 1973 and 1982, Salmonella Typhimurium was the most common Salmonella isolated from patients attending the University Hospital in Kuala Lumpur, Malaysia. Out of a total of 969 strains isolated from blood and feces (excluding S. Typhi), $155(\sim 16 \%)$ were Salmonella Typhimurium (S.D. Puthucheary, personal communication). Salmonella Typhimurium was also the most common NTS (10\%) isolated throughout Malaysia during the same decade [34] but dropped to $4.06 \%$ from 1983 to 1992 .

The occurrence of Salmonella Typhimurium phage type 104 (DT104) resistant to ampicillin, chloramphenicol, streptomycin, sulfonamide, and tetracycline has been reported worldwide [29]. The resistance of this serovar to cephalosporins, due to the production of extended-spectrum $\beta$-lactamase (ESBL), has also been reported [26]. Dissemination of the antimicrobial resistance is often through mobile genetic elements such as plasmids, transposons, and gene cassettes in integrons [20]. Class 1 integrons are most frequently found among clinical isolates that contain antimicrobial resistance genes in the internal variable region. This integron-containing antibiotic resistance gene cluster has been found near the 3' end of Salmonella genomic island 1 (SGI1) of serovar Typhimurium [8].

Phenotypic methods for identifying and typing of Salmonella Typhimurium are serotyping, biotyping, and phage typing, all showing limited discriminatory powers. Plasmid profiling, ribotyping, IS200 typing, multilocus 
sequence typing (MLST), PCR-based methods, and pulsedfield gel electrophoresis (PFGE) have recently proven to be useful in discriminating strains of $S$. Typhimurium $[13,32]$.

There is a lack of information on $S$. Typhimurium in Malaysia and this study was carried out to obtain better strain discrimination of this serovar isolated between 1969 to 2006 from animals and humans through a combination of methods. The detection and analysis of antibiotic resistance genes and integrons were carried out by using PCR and DNA sequencing, and the genetic relatedness was determined by PFGE and plasmid fingerprinting.

\section{Materials AND MethodS}

\section{Bacterial Strains}

Forty-seven Salmonella Typhimurium strains were studied. The human strains $(n=14)$ isolated from 1969 to 2006 were from a local teaching hospital. The zoonotic strains $(n=33)$ recovered from a variety of animals (cattle, $n=9$; chicken, $n=14$; swine, $n=1$; fish, $\mathrm{n}=1$; frog, $\mathrm{n}=1)$ and unknown zoonotic hosts $(\mathrm{n}=7)$ were provided by the Veterinary Research Institute, Malaysia. All the strains were confirmed to be $S$. Typhimurium by standard biochemical and serological tests at the Microbiology Laboratories of the Institutions that processed the specimens. The purity of the strains was checked by plating the cultures on selective media (XLD and BSA) at the Biomedical Science Laboratory, IPS, University of Malaya, where the analysis was carried out.

\section{Antimicrobial Susceptibility Testing}

Antimicrobial susceptibility testing was done using the disk diffusion assay on Mueller-Hinton agar with commercial antibiotic disks (Oxoid Ltd, Basingstoke, U.K.) according to the CLSI guidelines [11]. Escherichia coli ATCC 25922 and Pseudomonas aeruginosa ATCC 27853 were used as controls. The antimicrobials used were nalidixic acid $(10 \mu \mathrm{g})$, ampicillin $(10 \mu \mathrm{g})$, streptomycin $(10 \mu \mathrm{g})$, ciprofloxacin $(5 \mu \mathrm{g})$, trimethoprim/sulfamethoxazole $(25 \mu \mathrm{g})$, tetracycline $(30 \mu \mathrm{g})$, chloramphenicol $(30 \mu \mathrm{g})$, trimethoprim $(5 \mu \mathrm{g})$, gentamicin $(30 \mu \mathrm{g})$, kanamycin $(30 \mu \mathrm{g})$, sulfonamide $(300 \mu \mathrm{g})$, amoxicillin/ clavulanic acid $(30 \mu \mathrm{g})$, ceftiofur $(30 \mu \mathrm{g})$, cefepime $(30 \mu \mathrm{g})$, aztreonam $(30 \mu \mathrm{g})$, cephalothin $(30 \mu \mathrm{g})$, ceftazidime $(30 \mu \mathrm{g})$, cephradine $(30 \mu \mathrm{g})$, ceftriaxone $(30 \mu \mathrm{g})$, cephalexin $(30 \mu \mathrm{g})$, cefuroxime $(30 \mu \mathrm{g})$, and cefotaxime (30 $\mu \mathrm{g})$ (Becton Dickinson). Interpretation of inhibition zones was according to CLSI guidelines [11]. Interpretations for ceftiofur, which was not given in the CLSI guidelines, was as recommended by the manufacturer.

\section{DNA Template Preparation for PCR}

One bacterial colony from Luria-Bertani (LB) plate was suspended in $1.0 \mathrm{ml}$ of phosphate-buffered saline (PBS), centrifuged, and the cell pellet resuspended in $100 \mu \mathrm{l}$ of TE buffer $(10 \mathrm{mM}$ Tris, $1 \mathrm{mM}$ EDTA buffer, $\mathrm{pH} 8.0$ ) and boiled at $99^{\circ} \mathrm{C}$ for $10 \mathrm{~min}$. For each PCR reaction, $5 \mu \mathrm{l}$ ( $\sim 100 \mathrm{ng})$ of the supernatant was used.

\section{Differentiation of Phage Type DT104}

As phage typing facilities are not available in Malaysia, further differentiation of $S$. Typhimurium to phage type DT104, a commonly reported phage type associated with zoonotic strains, was performed using a multiplex PCR. Published primers for confirmation of Salmonella genus, Salmonella Typhimurium, and phage type DT104 are indicated in Table 1. PCR conditions consisted of an initial denaturation at $95^{\circ} \mathrm{C}$ for $2 \mathrm{~min}, 30$ cycles each of $95^{\circ} \mathrm{C}$ for $1 \mathrm{~min}$, $57^{\circ} \mathrm{C}$ for $1 \mathrm{~min}$, and $72^{\circ} \mathrm{C}$ for $2 \mathrm{~min}$, and a final extension at $72^{\circ} \mathrm{C}$ for $5 \mathrm{~min}$ [4].

\section{Detection of Resistance Genes}

PCR detection and confirmation of resistance to ampicillin, streptomycin, sulfonamides, and tetracycline were carried out using published primer sequences (Table 1). Initially, a monoplex-PCR was used for each of the primer sets to determine its specificity and reproducibility. Then, 3 multiplex-PCRs ( $\mathrm{mPCR}$ ) were optimized to enable simultaneous amplification of multiple resistance genes. mPCRI consisted of 4 primer pairs for bla $a_{\mathrm{TEM}}, s t r A$, sull, and sul2. mPCRII consisted of another 4 primer pairs for tet $A, \operatorname{tet} B, \operatorname{tem} B$, and aadA, whereas mPCRIII consisted of 3 primer pairs for tem $A, \operatorname{tet} G$, and tetC. The conditions for $\mathrm{mPCRI}$ were an initial denaturation at $94^{\circ} \mathrm{C}$ for $3 \mathrm{~min}$ followed by 30 cycles of $94^{\circ} \mathrm{C}$ for $30 \mathrm{~s}, 53^{\circ} \mathrm{C}$ for $30 \mathrm{~s}$, and $72^{\circ} \mathrm{C}$ for $1 \mathrm{~min}$, with a single final extension at $72^{\circ} \mathrm{C}$ for $7 \mathrm{~min}$. For $\mathrm{mPCRII}$ and $\mathrm{mPCRIII}$, the annealing conditions were changed to $62^{\circ} \mathrm{C}$ for $30 \mathrm{~s}$. All mPCRs were performed in a $25-\mu \mathrm{l}$ reaction mixture containing $1 \times$ PCR buffer, $1.5 \mathrm{mM} \mathrm{MgCl}_{2}, 200 \mu \mathrm{M}$ each of dNTPs, 60 pmol of each primer, $1 \mathrm{U}$ of Taq DNA polymerase (Intron, Biotechnology, South Korea), and 100 ng of template DNA. The PCR products were analyzed by electrophoresis through a $1.5 \%$ agarose gel (Promega, Madison, U.S.A), staining with ethidium bromide $(0.5 \mu \mathrm{g} / \mathrm{ml})$, and then visualizing using the GelDoc (BioRad). A DNA ladder of $100 \mathrm{bp}$ (Promega) was used as the molecular weight marker.

\section{Detection of Integrons}

Isolates were screened for the presence of class 1 integrons using specific primers previously described [20] to anneal the $5^{\prime}$ conserved sequences (CS) and 3' CS flanking the integrated gene cassettes (Table 1). PCR was performed in a $25-\mu$ l final volume containing $1 \times$ PCR buffer, $1.5 \mathrm{mM} \mathrm{MgCl} 2,200 \mu \mathrm{M}$ each of dNTPs, $1 \mathrm{U}$ Taq DNA polymerase (Intron, Biotechhnology, South Korea), 25 pmol of each primer, and $100 \mathrm{ng}$ of template DNA. The cycling conditions consisted of an initial denaturation at $94^{\circ} \mathrm{C} 10 \mathrm{~min}, 35$ cycles each of $94^{\circ} \mathrm{C}$ for $1 \mathrm{~min}, 55^{\circ} \mathrm{C}$ for $1 \mathrm{~min}$, and $72^{\circ} \mathrm{C}$ for $5 \mathrm{~min}$, and further extension at $72^{\circ} \mathrm{C}$ for $5 \mathrm{~min}$ [12]. The amplification reaction included a negative control, which contained all reagents except target DNA. The PCR products were analyzed by electrophoresis on $1 \%$ agarose gel.

\section{DNA Sequencing}

Selected amplified DNA products were verified by DNA sequencing. The amplicons were purified using a DNA purification kit (Qiagen, Germany) and sent to a commercial facility for sequencing (Research Biolabs, Singapore). The ABI PRISM Big Dye terminator cycle sequencing system was used (Perkin Elmer, Applied Biosystems). The resulting DNA sequence data were compared with the GenBank database using the BLAST algorithm available on the NCBI Web site (http://www.ncbi.nih.gov).

\section{Plasmid Profiling}

Plasmid DNA was extracted using a Q1Aprep spin miniprep kit (Qiagen, Germany) according to the manufacturer's recommendation. The plasmid DNA was analyzed through $0.8 \%$ horizontal agarose 
Table 1. Primers for detection of resistance genes and integrons in Salmonella via PCR.

\begin{tabular}{|c|c|c|c|c|}
\hline Primers names & Sequence $\left(5^{\prime}-3^{\prime}\right)$ & Amplification target & Size (bp) & Reference \\
\hline $\begin{array}{l}\text { OMPCF } \\
\text { OMPCR }\end{array}$ & $\begin{array}{l}\text { ATCGCTGACTTATGCAATCG } \\
\text { CGGGTTGCGTTATAGGTCGT }\end{array}$ & Salmonella genus & 204 & [4] \\
\hline $\begin{array}{l}104 \mathrm{~F} \\
104 \mathrm{R}\end{array}$ & $\begin{array}{l}\text { ATGCGTTTGGTCTCAACGCC } \\
\text { GCTGAGGCCACGGATATTTA }\end{array}$ & $\begin{array}{l}\text { S. Typhimurium } \\
\text { DT104 }\end{array}$ & 102 & [4] \\
\hline $\begin{array}{l}\text { TyphF } \\
\text { TyphR }\end{array}$ & $\begin{array}{l}\text { TTGTTCACTTTTTACCCCTGAA } \\
\text { CCCTGACAGCCGTTAGATATT }\end{array}$ & $S$. Typhimurium & 401 & [4] \\
\hline $5^{\prime} \mathrm{CS} 3^{\prime} \mathrm{CS}$ & $\begin{array}{l}\text { GGCATCCAAGCAGCAAG } \\
\text { AAGCAGACTTGACCTGA }\end{array}$ & Integron & Variable & [20] \\
\hline blaTem & $\begin{array}{l}\text { ATGAGTATTCAACATTTCCG } \\
\text { ACCAATGCTTAATCAGTGAG }\end{array}$ & $b l a_{\mathrm{TEM}}$ & 859 & [2] \\
\hline StrA & $\begin{array}{l}\text { CCAATCGCAGATAGAAGGC } \\
\text { CTTGGTGATAACGGCAATTC }\end{array}$ & strA & 548 & [2] \\
\hline Sul1 & $\begin{array}{l}\text { CTTCGATGAGAGCCGGCGGC } \\
\text { GCAAGGCGGAAACCCGCGCC }\end{array}$ & sull & 435 & [2] \\
\hline Sul2 & $\begin{array}{l}\text { GCGCTCAAGGCAGATGGCATT } \\
\text { GCGTTTGATACCGGCACCCGT }\end{array}$ & sul2 & 293 & [2] \\
\hline tem A & $\begin{array}{l}\text { ATGAGTATTCAACATTTCCG } \\
\text { CTGACAGTTACCAATGCTTA }\end{array}$ & temA & 867 & [23] \\
\hline temB & $\begin{array}{l}\text { TTTTCGTGTCGCCCTTATTCC } \\
\text { CGTTCATCCATAGTTGCCTGACTC }\end{array}$ & $\operatorname{tem} B$ & 79 & [31] \\
\hline TetA & $\begin{array}{l}\text { GTAATTCTGAGCACTGTCGC } \\
\text { CTGCCTGGACAACATTGCTT }\end{array}$ & tetA & 956 & [2] \\
\hline TetB & $\begin{array}{l}\text { CTCAGTATTCCAAGCCTTTG } \\
\text { ACTCCCCTGAGCTTGAGGGG }\end{array}$ & $\operatorname{tet} B$ & 414 & [2] \\
\hline TetC & $\begin{array}{l}\text { GGTTGAAGGCTCTCAAGGGC } \\
\text { CCTCTTGCGGGAATCGTCC }\end{array}$ & tet $C$ & 505 & [2] \\
\hline TetG & $\begin{array}{l}\text { GCAGCGAAAGCGTATTTGCG } \\
\text { TCCGAAAGCTGTCCAAGCAT }\end{array}$ & $\operatorname{tet} G$ & 662 & [2] \\
\hline aadA & $\begin{array}{l}\text { ATCCTTCGGCGCGATTTTG } \\
\text { GCAGCGCAATGACATTCTTG }\end{array}$ & aadA & 282 & [2] \\
\hline
\end{tabular}

gel electrophoresis. E. coli $39 \mathrm{R}$ and E. coli V517 were used as molecular weight markers.

\section{PFGE Analysis}

PFGE was carried out according to a previous protocol [28]. DNA was digested with $10 \mathrm{U}$ of restriction enzyme Xbal (5'-TCTAGA-3') (Promega, Madison, WI, U.S.A.) at $37^{\circ} \mathrm{C}$. The restriction fragments were separated by electrophoresis in $0.5 \times$ TBE buffer, for $22 \mathrm{~h}$ at $14^{\circ} \mathrm{C}$ in a CHEF Mapper system (Bio-Rad, U.S.A.) using pulsed times of 2.2 to 63.8 s. XbaI-digested S. Braenderup H9812 was used as the DNA size marker. PFGE data were analyzed using Gel Compar software (version 4, Applied Maths). The extent of variability was determined by the Dice coefficient F, as previously described [28]. Clustering was based on the unweighted pair group average method (UPGMA) with a position tolerance of 0.10 .

\section{RESUlts}

\section{PCR and Phage types}

The multiplex PCR products consisted of a 204-bp fragment of the ompC sequence that is specific for the Salmonella genus, a 401-bp fragment of the specific sequence in Typhimurium, and a 102-bp fragment of the specific sequence in DT104. Out of the initial $59 \mathrm{~S}$. Typhimurium isolates provided by the collaborating centers, 47 were confirmed as serotype Salmonella Typhimurium as indicated by the presence of 2 bands, $204 \mathrm{bp}$ and $401 \mathrm{bp}$. The other 12 Salmonella strains had only one amplicon, $204 \mathrm{bp}$. Among the $47 \mathrm{~S}$. Typhimurium strains, 20 were phage type DT104.

\section{Antibiograms}

Only 9 of the 47 confirmed $S$. Typhimurium strains were susceptible to all the 22 antimicrobials tested, whereas 33 $(70.2 \%)$ were resistant to more than one. All the animal strains were susceptible to ciprofloxacin, cefuroxime, ceftazidime, and aztreonam, whereas the human strains were resistant to at least one antimicrobial agent (Table 2). High rates of resistance to tetracycline $(70.2 \%)$, sulfonamide (57.4\%), streptomycin (53.1\%), ampicillin (29.7\%), nalidixic acid (27.6\%), kanamycin (23.4\%), chloramphenicol (21.2\%), and trimethoprim (19.1\%) were observed for both human and zoonotic strains (Table 2). Resistance towards cephalosporins 
Table 2. Antimicrobial resistance among $S$. Typhimurium strains.

\begin{tabular}{lccc}
\hline \multirow{2}{*}{ Antimicrobial agents } & \multicolumn{3}{c}{ Numbers (\%) of resistant strains } \\
\cline { 2 - 4 } & $\begin{array}{c}\text { Animal } \\
(\mathrm{n}=33)\end{array}$ & $\begin{array}{c}\text { Human } \\
(\mathrm{n}=14)\end{array}$ & Total \\
\hline Ampicillin & $8(24)$ & $6(43)$ & $14(30)$ \\
Chloramphenicol & $5(15)$ & $5(36)$ & $10(21)$ \\
Ciprofloxacin & $0(0)$ & $1(7)$ & $1(2)$ \\
Gentamicin & $4(12)$ & $1(7)$ & $5(11)$ \\
Kanamycin & $5(15)$ & $6(43)$ & $11(23)$ \\
Streptomycin & $15(45)$ & $10(71)$ & $25(53)$ \\
Nalidixic acid & $12(36)$ & $1(7)$ & $13(28)$ \\
Sulfonamides & $16(48)$ & $11(79)$ & $27(57)$ \\
Trimethoprim & $5(15)$ & $4(29)$ & $9(19)$ \\
Sxt & $4(12)$ & $2(14)$ & $6(15)$ \\
Tetracycline & $21(64)$ & $12(86)$ & $33(70)$ \\
Cephradine & $5(15)$ & $5(36)$ & $10(21)$ \\
Cephalexin & $2(6)$ & $6(43)$ & $8(17)$ \\
Ceftriaxone & $1(3)$ & $1(7)$ & $2(4)$ \\
Cefuroxime & $0(0)$ & $1(7)$ & $1(2)$ \\
Cephalothin & $7(21)$ & $6(43)$ & $13(28)$ \\
Ceftazidime & $0(0)$ & $1(75)$ & $1(2)$ \\
AMC & $4(12)$ & $4(29)$ & $8(17)$ \\
Cefepime & $0(0)$ & $1(7)$ & $1(2)$ \\
Cefotaxime & $1(3)$ & $1(7)$ & $2(5)$ \\
Aztreonam & $0(0)$ & $1(7)$ & $1(2)$ \\
Ceftiofur & $0(0)$ & $1(7)$ & $1(2)$ \\
\hline
\end{tabular}

Sxt, trimethoprim/sulfamethoxazole; AMC, amoxicillin-clavulanic acid.

was as follows: cephalothin (27.6\%), cephradine (21.2\%), amoxicillin (17.0\%), and cephalexin (17.0\%). Twenty-nine resistotypes were defined (Table 3 ). The predominant resistotype was P8 (simultaneous resistance to $\mathrm{S}, \mathrm{Su}, \mathrm{T}$ ), followed by resistotypes $\mathrm{P} 3$ and $\mathrm{P} 7$. The rest of the resistotypes were represented by one strain each (Table 3 ).

Multidrug resistance (MDR), defined as resistance to 3 or more groups of antimicrobial agents, was detected in 27 (57.4\%) strains (Table 3). Three common R-types were observed: R-type ACSSuTe (n=8), R-type SSuTe $(n=7)$, and R-type KSSuTeTm (n=3) with or without additional resistance to antimicrobial agents.

\section{Detection of Resistance Genes}

The simultaneous detection of different antimicrobial resistance genes such as $b l a_{\text {TEM }}$, strA, aadA, sull, sul2, tetA, $\operatorname{tet} B, \operatorname{tet} C$, and $\operatorname{tet} G$ was carried out using three multiplexPCRs (Fig. 1). All the $14(100 \%)$ ampicillin-resistant strains harbored $b_{l a} a_{\mathrm{TEM}}, t e m A$, and temB with sizes $859 \mathrm{bp}, 867 \mathrm{bp}$, and $798 \mathrm{bp}$, respectively. A comparison between sequenced fragments of $b l a_{\mathrm{TEM}}$-positive strains and available sequences in the GenBank revealed similar identity $(95 \%)$ with bla $_{\mathrm{TEM}}$. All 25 streptomycin-resistant strains harbored the strA gene, and only $14(56.0 \%)$ harbored the aadA gene. Both str $A$ and aadA genes were present in $11(44.0 \%)$ strains. Ten of the $27(37.0 \%)$ sulfonamide-resistant strains harbored sul1, and 24 (88.8\%) harbored sul2, whereas 7 $(25.9 \%)$ strains had both sull and sul2. Of the 33 tetracycline-resistant strains, $23(69.6 \%)$ contained the tetA gene, $5(15.1 \%)$ had tet $B$, and $4(12.1 \%)$ had tetC. No strain was positive for tet $G$. A combination of tetA $A+t e t B$ genes was found in one strain (STM DT104 6344/95). One tetracycline-resistant clinical strain (STM 0287/69) did not harbor any of the 4 tetracycline-resistant genes.

\section{Detection of Integrons}

Class 1 integrons were present in 33 (70.2\%) of the strains: 13 clinical and 20 zoonotic. Three strains carried one integron, 19 strains carried 2, and 11 strains carried 3. All except one integron-positive strain carried the 210-bp size integron. Sequencing analysis showed that the 210-bp fragment is a part of the $p u r G$ gene that encodes the enzyme phosphoribosylformylglycinamidine synthetase (GenBank Accession No. AF151984). In the other profiles, 5 additional amplicons were detected (650 bp, $800 \mathrm{bp}, 1,000 \mathrm{bp}, 1,200 \mathrm{bp}$, $1,900 \mathrm{bp}$ ). Integron $650 \mathrm{bp}$ contained the sat gene that encodes the enzyme streptothricin acetyltransferase and confers resistance to streptothricin. Intergron $800 \mathrm{bp}$ harbored $d f r A 7$ that encodes the enzyme dihydrofolate reductase

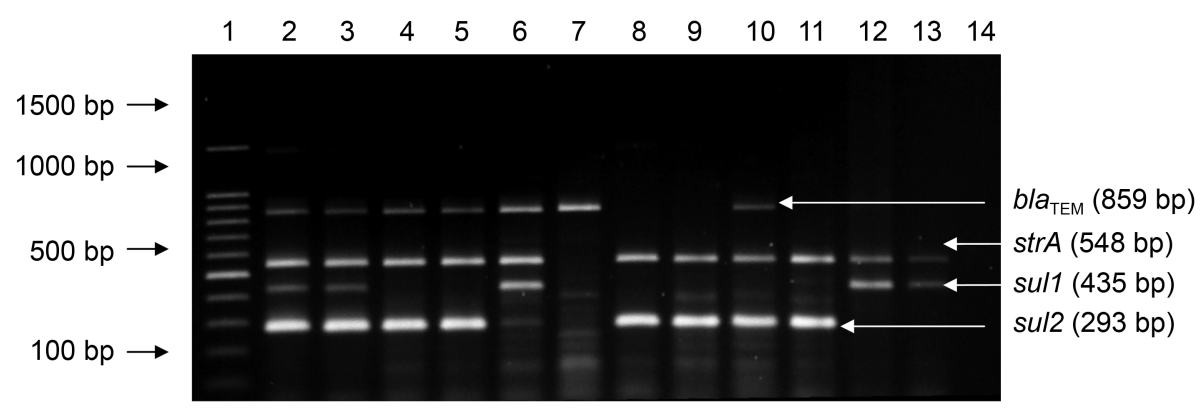

Fig. 1. Representative gel of multiplex-PCR for detection of resistance genes.

DNA bands are indicated by bla TEM $(859 \mathrm{bp})$, strA (548 bp), sull (435 bp), and sul2 (293 bp). Lanes 1-14: DNA size standard (100-bp ladder), positive control, 2625/05, 1204/05, 110187/70, 3866/05, 3865/05, 3077/05, 5229/05, 2559/05, 32/05, 447/05, 447/05, negative control. 
Table 3. Phenotypic and genotypic characteristics of zoonotic and human $S$. Typhimurium strains.

\begin{tabular}{|c|c|c|c|c|c|c|c|}
\hline Strain No. & Source & $\mathrm{PT}^{\mathrm{a}}$ & Resistotypes (profiles) & Resistance genes present & Plasmid sizes $(\mathrm{kb})$ & Integrons $(\mathrm{kb})$ & $\begin{array}{l}\text { PFGE } \\
\text { profiles }\end{array}$ \\
\hline $5553 / 04$ & Animal $^{\mathrm{c}}$ & nd & $\$$ & $\mathrm{~N}$ & 90 & $\mathrm{~N}$ & $\mathrm{X} 26$ \\
\hline $2593 / 04$ & Cattle & nd & $\$$ & $\mathrm{~N}$ & 90 & $\mathrm{~N}$ & $\mathrm{X} 16$ \\
\hline $1377 / 05$ & Frog & nd & $\$$ & $\mathrm{~N}$ & $2.0,28.9,95$ & $\mathrm{~N}$ & X39 \\
\hline $1621 / 05$ & Chicken & nd & $\$$ & $\mathrm{~N}$ & 90 & $\mathrm{~N}$ & $\mathrm{X} 8$ \\
\hline $2560 / 05$ & Chicken & nd & $\$$ & $\mathrm{~N}$ & $3.8,2.6$ & $\mathrm{~N}$ & $\mathrm{X} 30$ \\
\hline $2552 / 05$ & Cattle & nd & $\$$ & $\mathrm{~N}$ & $13,34.3,65.2$ & $\mathrm{~N}$ & $\mathrm{X} 37$ \\
\hline $3000 / 05$ & Fish & nd & $\$$ & $\mathrm{~N}$ & $13,34.3,65.2$ & $\mathrm{~N}$ & $\mathrm{X} 21$ \\
\hline $2553 / 05$ & Cattle & $104^{\mathrm{b}}$ & $\$$ & $\mathrm{~N}$ & $\mathrm{~N}$ & $\mathrm{~N}$ & $\mathrm{X} 22$ \\
\hline $0504 / 69$ & Human & 104 & $\$$ & $\mathrm{~N}$ & 2.7 & $\mathrm{~N}$ & $\mathrm{X} 7$ \\
\hline $3215 / 03$ & Cattle & 104 & $\mathrm{CE}(\mathrm{P} 1)$ & $\mathrm{N}$ & $\mathrm{N}$ & $\mathrm{N}$ & $\mathrm{X} 15$ \\
\hline $1234 / 04$ & Animal & nd & $\mathrm{Su}(\mathrm{P} 2)$ & sul2 & $\mathrm{N}$ & $0.21,0.6$ & $\mathrm{X} 26$ \\
\hline $402 / 05$ & Chicken & nd & $\mathrm{T}(\mathrm{P} 3)$ & tet $A$ & 90 & $0.21,0.8$ & $\mathrm{X} 17$ \\
\hline $2599 / 05$ & Animal & 104 & $\mathrm{~T}(\mathrm{P} 3)$ & tetA & $\mathrm{N}$ & $\mathrm{N}$ & $\mathrm{X} 25$ \\
\hline $3865 / 05$ & Chicken & 104 & $\mathrm{~A}, \mathrm{Nx}(\mathrm{P} 4)$ & $b l a_{\mathrm{TEM}}$ & $\mathrm{N}$ & $\mathrm{N}$ & $\mathrm{X} 30$ \\
\hline $01 / 06$ & Human & 104 & CE, CL (P5) & $\mathrm{N}$ & $\mathrm{N}$ & $0.21,0.8$ & X19 \\
\hline $7456 / 04$ & Cattle & 104 & $\mathrm{Su}, \mathrm{T}(\mathrm{P} 6)$ & sul2, tet $A$ & $\mathrm{~N}$ & $0.21,0.6$ & X9 \\
\hline $5067 / 05$ & Animal & nd & $\mathrm{Nx}, \mathrm{T}(\mathrm{P} 7)$ & tetA & $\mathrm{N}$ & $0.21,0.6$ & $\mathrm{X} 27$ \\
\hline $3503 / 05$ & Chicken & nd & Nx, T (P7) & tetA & $\mathrm{N}$ & $\mathrm{N}$ & $\mathrm{X} 11$ \\
\hline $2555 / 05$ & Cattle & 104 & Nx, T (P7) & tetA & $\mathrm{N}$ & $\mathrm{N}$ & $\mathrm{X} 10$ \\
\hline $8322 / 03$ & Chicken & nd & $\mathrm{S}, \mathrm{Su}, \mathrm{T}(\mathrm{P} 8)$ & str $A$, sul2, tet $A$ & $11.3,22.8,50$ & $0.21,1.0$ & $\mathrm{X} 28$ \\
\hline $6324 / 03$ & Chicken & nd & $\mathrm{S}, \mathrm{Su}, \mathrm{T}(\mathrm{P} 8)$ & str $A$, sul2, tet $A$ & $11.3,22.8,50,90$ & $0.21,1.0$ & $\mathrm{X} 28$ \\
\hline $4524 / 05$ & Chicken & nd & $\mathrm{S}, \mathrm{Su}, \mathrm{T}(\mathrm{P} 8)$ & str $A$, sul2, tet $A$ & $3.1,11.3,22.8,50$ & $0.21,1.0$ & $\mathrm{X} 31$ \\
\hline $0287 / 69$ & Human & 104 & $\mathrm{~S}, \mathrm{Su}, \mathrm{T}(\mathrm{P} 8)$ & strA, sull, sul2 & $11.3,22.8,50$ & $0.21,1.0$ & $\mathrm{X} 3$ \\
\hline $113254 / 70$ & Human & 104 & $\mathrm{~S}, \mathrm{Su}, \mathrm{T}(\mathrm{P} 8)$ & str $A$, sul2, tetA & $2.6,11.3,22.8$ & $0.21,1.0$ & $\mathrm{X} 1$ \\
\hline $32 / 05$ & Human & nd & $\mathrm{S}, \mathrm{Su}, \mathrm{T}(\mathrm{P} 8)$ & str $A$, sul2, tetA & $11.3,22.8,50$ & $0.21,1.0$ & $\mathrm{X} 18$ \\
\hline $254 / 98$ & Chicken & nd & G, Nx, S, T (P9) & $\operatorname{str} A, \operatorname{tet} B$ & 5.4 & $0.21,1.0$ & $\mathrm{X} 12$ \\
\hline $981 / 04$ & Animal & nd & $\mathrm{Nx}, \mathrm{S}, \mathrm{Su}, \mathrm{T}(\mathrm{P} 10)$ & str $A$, sul2, tetA & $22.8,50,90$ & $0.21,1.0$ & $\mathrm{X} 36$ \\
\hline $5229 / 05$ & Chicken & nd & $\mathrm{K}, \mathrm{S}, \mathrm{Su}, \mathrm{T}, \mathrm{Tm}(\mathrm{P} 13)$ & strA, sul2, tetA & $11.3,22.8,50,90$ & $0.21,0.6,1.0$ & $\mathrm{X} 27$ \\
\hline $447 / 05$ & Human & 104 & $\mathrm{~K}, \mathrm{~S}, \mathrm{Su}, \mathrm{Sxt}, \mathrm{T}, \mathrm{Tm}(\mathrm{P} 14)$ & str $A$, sull, tetA, aadA & $3.4,4.3,90$ & $0.21,1.9$ & $\mathrm{X} 24$ \\
\hline
\end{tabular}




\begin{tabular}{|c|c|c|c|c|c|c|c|}
\hline Strain No. & Source & $\mathrm{PT}^{\mathrm{a}}$ & Resistotypes (profiles) & Resistance genes present & Plasmid sizes $(\mathrm{kb})$ & Integrons $(\mathrm{kb})$ & $\begin{array}{l}\text { PFGE } \\
\text { profiles }\end{array}$ \\
\hline $87098 / 70$ & Human & 104 & CE, CL, KF, S, Su, T (P15) & str $A$, sul2, tet $A$, aad $A$ & $11.3,22.8,50,90$ & $0.21,0.6,1.0$ & $\mathrm{X} 1$ \\
\hline $6344 / 95$ & Animal & 104 & C, CE, AMC, KF, S, Su, T (P16) & str $A$, sul1, sul2, tet $A$, tetB, aadA & $11.3,22.8,50,90$ & $0.21,1.0,1.2$ & $\mathrm{X} 14$ \\
\hline $5532 / 04$ & Animal & nd & $\begin{array}{l}\mathrm{A}, \mathrm{N}, \mathrm{Su}, \mathrm{KF}, \mathrm{CE}, \mathrm{CL} \\
\mathrm{AMC}(\mathrm{P} 17)\end{array}$ & $b l a_{\mathrm{TEM}}$, sul2 & $2.0,2.2$ & $0.21,0.6$ & $\mathrm{X} 35$ \\
\hline $3866 / 05$ & Chicken & nd & A, Nx, S, Su, Sxt, T, Tm (P18) & bla $_{\mathrm{TEM}}$, str $A$, sull tet $A$, temB, aadA, tem $A$ & $2.2,3.4$ & $1.2,1.9$ & $\mathrm{X} 38$ \\
\hline $3077 / 05$ & Swine & nd & $\begin{array}{l}\mathrm{K}, \mathrm{S}, \mathrm{Su}, \mathrm{T}, \mathrm{Tm}, \mathrm{AMC} \\
\mathrm{KF}, \mathrm{CE}(\mathrm{P} 19)\end{array}$ & str $A$, sul2, tetA & $10.6,22.8$ & $0.21,0.6,1.0$ & X34 \\
\hline $95893 / 70$ & Human & 104 & $\mathrm{~A}, \mathrm{C}, \mathrm{S}, \mathrm{Su}, \mathrm{T}, \mathrm{K}, \mathrm{KF}, \mathrm{CL}(\mathrm{P} 20)$ & $\begin{array}{l}\text { bla }_{\mathrm{TEM}}, \text { strA, sull, sul2, temB, aadA, } \\
\text { temA, tetC }\end{array}$ & $2.6,3.0,7.5,11.3,32.1,90$ & $0.21,1.0,1.2$ & $\mathrm{X} 5$ \\
\hline $79495 / 70$ & Human & 104 & $\begin{array}{l}\text { A, C, S, Su, T, AMC, K, KF } \\
(\mathrm{P} 21)\end{array}$ & $\begin{array}{l}\text { bla } \\
\text { tetC }\end{array}$ & $34.3,90$ & $0.21,1.0,1.2$ & $\mathrm{X} 1$ \\
\hline $30822 / 70$ & Human & 104 & $\begin{array}{l}\text { A, C, K, Su, T, AMC, KF, } \\
\text { CL (P22) }\end{array}$ & $\begin{array}{l}\text { bla } a_{\mathrm{TEM}}, \text { sull, sul2, temB, aadA, temA, } \\
\text { tetC }\end{array}$ & $32.3,90$ & $0.21,1.0$ & $\mathrm{X} 2$ \\
\hline $3079 / 05$ & Cattle & nd & $\begin{array}{l}\text { A C, S, Su, T, K, Nx, Sxt, } \\
\text { Tm, KF (P23) }\end{array}$ & $\begin{array}{l}\text { bla } a_{\mathrm{TEM}}, \text { str } A, \text { sull, sul2, tet } A, \operatorname{tem} B \\
\operatorname{aad} A, \text { temA }\end{array}$ & $11.3,22.8,50,90$ & $0.21,0.6,1.9$ & $\mathrm{X} 29$ \\
\hline $2559 / 05$ & Cattle & nd & $\begin{array}{l}\text { A, C, S, Su, T, Nx, Sxt, Tm, } \\
\text { G, KF (P24) }\end{array}$ & $b^{b l a} a_{\mathrm{TEM}}$, str $A$, sul2, temB, aadA, tetB & $4.9,83.4,90$ & 0.21 & $\mathrm{X} 13$ \\
\hline $2625 / 05$ & Chicken & nd & $\begin{array}{l}\text { A, C, S, Su, T, K, Nx, Sxt, } \\
\text { Tm, AMC, KF (P25) }\end{array}$ & $\begin{array}{l}\text { bla } \mathrm{T}_{\mathrm{TEM}}, \text { strA, sull, sul2, tetA, temB, } \\
\text { aadA, temA }\end{array}$ & $10.6,28.3,52.6$ & $0.21,0.6,1.9$ & $\mathrm{X} 20$ \\
\hline $110187 / 70$ & Human & 104 & $\begin{array}{l}\text { A, C, S, Su, T, K, Tm, AMC, } \\
\text { KF, CE, CL (P26) }\end{array}$ & $b l a_{\mathrm{TEM}}, \operatorname{str} A, \operatorname{sul} 2, \operatorname{tem} B$, aad $A, \operatorname{tem} A$ & $34.3,90$ & $0.21,0.6,1.0$ & $\mathrm{X} 4$ \\
\hline $02 / 06$ & Human & nd & $\begin{array}{l}\text { A, C, S, Su, T, Cip, G, K, Nx, } \\
\text { Sxt, Tm (P27) }\end{array}$ & $\begin{array}{l}\text { bla } a_{\mathrm{TEM}}, \text { str } A, \text { sull, tet } A, \text { tem } B, \\
\text { aadA, tem } A\end{array}$ & $2.6,3.0,7.5,11.3,22.8,50$ & $0.21,0.6,1.9$ & $\mathrm{X} 23$ \\
\hline $1204 / 05$ & Chicken & 104 & $\begin{array}{l}\text { A, C, S, Su, T, G, K, KF, } \\
\text { AMC, CRO, CTX, CE, CL } \\
(\mathrm{P} 28)\end{array}$ & $b l a_{\mathrm{TEM}}$, str $A$, sul2, tet $A$, tem $A$ & $3.4,90$ & 0.21 & X33 \\
\hline 196/05 & Human & nd & $\begin{array}{l}\text { A, S, Su, T, XNL, KF, CL, } \\
\text { CE, CXM, CRO, CTX, CAZ, } \\
\text { AMC, FEP, ATM (P29) }\end{array}$ & $b l a_{\mathrm{TEM}}$, strA, sul2, tetA, temB & $3.4,4.3,11.3,22.8,50$ & $0.21,1.0,1.2$ & $\mathrm{X} 18$ \\
\hline
\end{tabular}


and confers resistance to trimethoprim. Integron $1,000 \mathrm{bp}$ harbored the aadA2 gene that encodes for aminoglycoside adenyltransferase $\mathrm{AAD}\left(3^{\prime \prime}\right)$ and confers resistance to streptomycin-spectinomycin. Integron 1,200 bp harbored the $p s e-1$ gene that encodes for beta-lactamase and confers resistance to ampicillin. Integron $1,900 \mathrm{bp}$ harbored the dfrA12 gene located near the 5 ' end that encodes for dihydrofolate reductase and confers resistance to trimethoprim, and the $\operatorname{aad} A 2$ gene located near the 3 ' end that encodes aminoglycoside adenyltransferase $\operatorname{AAD}\left(3^{\prime \prime}\right)$ and confers resistance to streptomycin-spectinomycin.

\section{Plasmid Analysis}

Thirty-five (74.4\%) strains (12 human, 23 animal) harbored 1-6 plasmids ( 2.0 to $95 \mathrm{~kb}$ ) each (Table 3$)$. Two human strains (STM 02/06 and STM 95893/70) had 6 plasmids (Table 3). The predominant plasmid was $90 \mathrm{~kb}$, present in $17(48.5 \%)$ strains, followed by $22.8 \mathrm{~kb}(\mathrm{n}=14), 11.3 \mathrm{~kb}$ $(\mathrm{n}=13)$, and $50 \mathrm{~kb}(\mathrm{n}=12)$.

\section{Genotyping}

PFGE of XbaI-digested chromosomal DNA subtyped all the 47 strains into 39 pulsotypes (PFPs) with DNA fragments

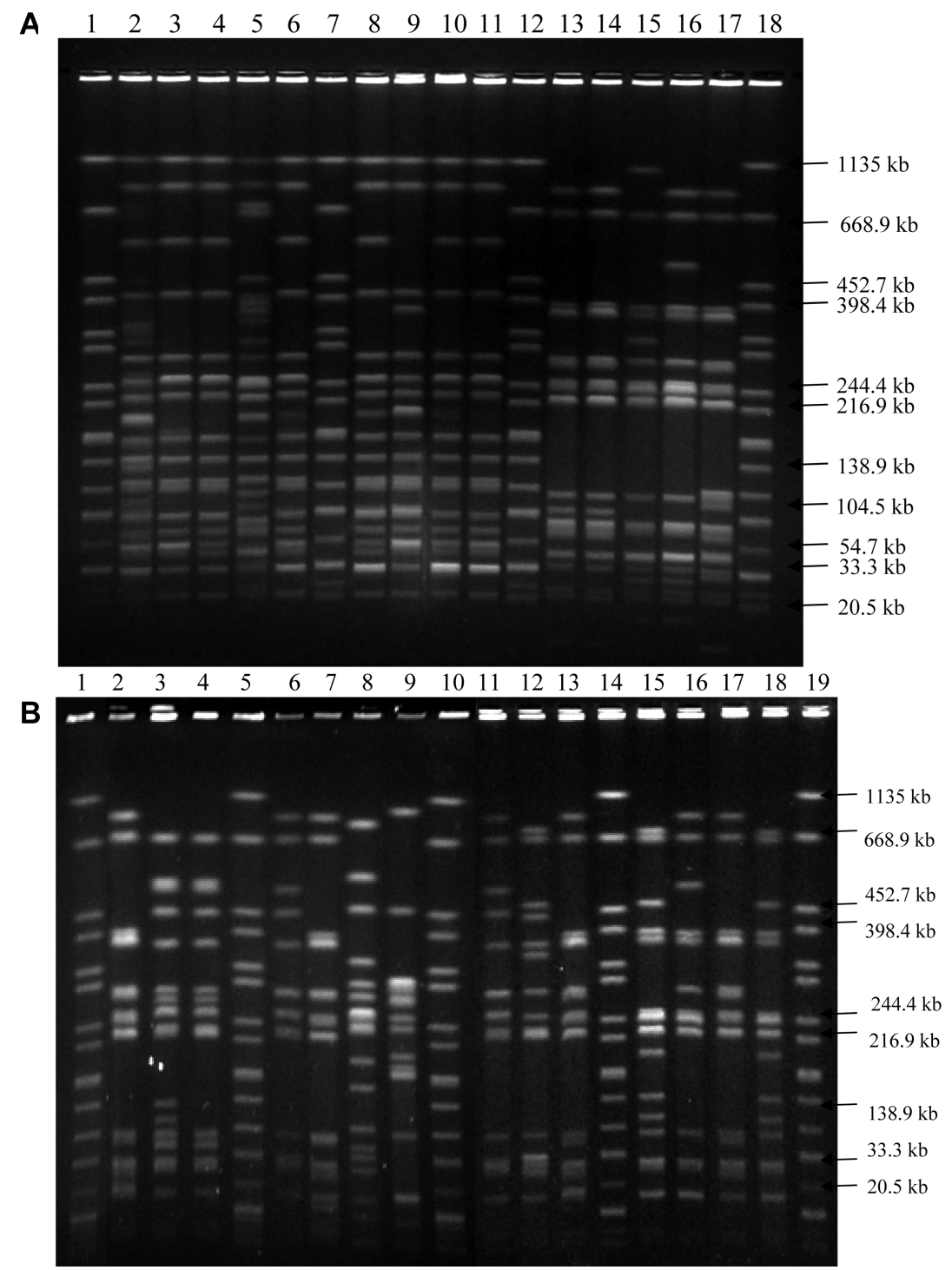

Fig. 2. PFGE profiles of selected human and zoonotic $S$. Typhimurium strains.

A. Representative XbaI-PFGE profiles of selected human $S$. Typhimurium strains. Lanes 1, 7, 12, 18: Salmonella H9812 marker strain; lanes 2-6, 8-11, 13-17: 95893/70, 110187/70, 13254/70, 0504/69, 0287/69, 30822/70, 28723/77, 87098/70, 79495/70, 32/05, 196/0, 447/05, 01/06, 02/06. B. Representative XbaI-PFGE profiles of selected zoonotic $S$. Typhimurium strains. Lanes 1, 5, 10, 14, 19: Salmonella H9812 DNA marker strain; lanes 2-4, 6-9, 11-13, 1518: 6321/03, 981/04, 5532/04,1234/04, 3079/05, 3503/05, 3866/05, 5553/04, 2593/04, 5231/05, 2560/05, 2553/05, 3000/05, $3865 / 05$. 


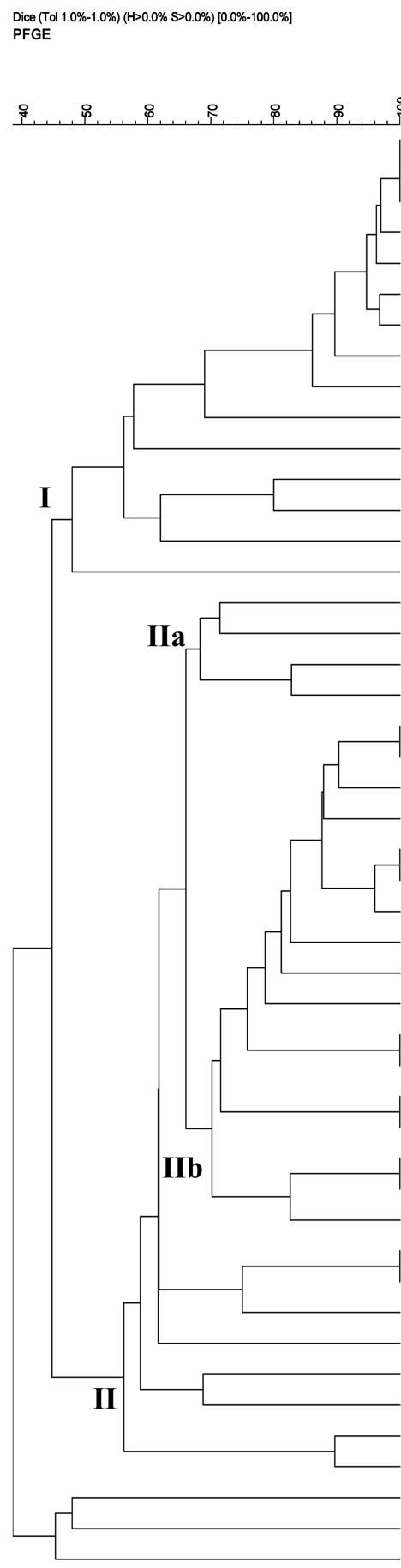

Strains
Code $\quad$ Source $\quad$ Phagetype $\underset{\text { Profile }}{\text { PFGE }}$

\begin{tabular}{|c|c|c|c|}
\hline STM 113254/70 & Human & DT104 & $\mathrm{X} 1$ \\
\hline STM 87098/70 & Human & DT104 & $\mathrm{x} 1$ \\
\hline STM 79495/70 & Human & DT104 & $\mathrm{x} 1$ \\
\hline STM 30822/70 & Human & DT104 & X2 \\
\hline STM $0287 / 69$ & Human & DT104 & $x_{3}$ \\
\hline STM $110187 / 70$ & Human & DT104 & $\mathrm{x} 4$ \\
\hline STM 95893/70 & Human & DT104 & $\times 5$ \\
\hline STM 287232/77 & Human & $\mathrm{n}$ & $\mathrm{x} 6$ \\
\hline STM 0504/69 & Human & DT104 & $x 7$ \\
\hline STM 1621/05 & Chicken & $\mathrm{n}$ & x8 \\
\hline STM 7456/04 & Cattle & DT104 & $\times 9$ \\
\hline STM 2555/05 & Cattle & DT104 & $\mathrm{X} 10$ \\
\hline STM 3503/05 & Duck & $n$ & $\mathrm{X} 11$ \\
\hline STM 254/98 & Chicken & $\mathrm{n}$ & $\mathrm{X} 12$ \\
\hline STM 2559/05 & Cattle & $\mathrm{n}$ & $\mathrm{X} 13$ \\
\hline STM 6344/95 & Zoonotic & DT104 & $\mathrm{X} 14$ \\
\hline STM 3215/03 & Cattle & DT104 & $\mathrm{X} 15$ \\
\hline STM 2593/04 & Cattle & $\mathrm{n}$ & $\mathrm{X} 16$ \\
\hline STM 402/05 & Chicken & $n$ & $\mathrm{X} 17$ \\
\hline STM TA196/05 & Human & $\mathrm{n}$ & $\mathrm{X} 18$ \\
\hline STM TA32/05 & Human & $\mathrm{n}$ & $\mathrm{X} 18$ \\
\hline STM 01/06 & Human & DT104 & $\mathrm{X} 19$ \\
\hline STM 2625/05 & Chicken & $\mathrm{n}$ & $\times 20$ \\
\hline STM 5231/05 & Cattle & DT104 & $\mathrm{X} 21$ \\
\hline STM 3000/05 & Fish & $\mathrm{n}$ & X21 \\
\hline STM 2553/05 & Cattle & DT104 & $\mathrm{X} 22$ \\
\hline STM 02/06 & Human & $\mathrm{n}$ & $\mathrm{x} 23$ \\
\hline STM TA447/05 & Human & DT104 & X24 \\
\hline STM 2599/05 & Zoonotic & DT104 & $\times 25$ \\
\hline STM 5553/04 & Zoonotic & $\mathrm{n}$ & $\times 26$ \\
\hline STM 1234/04 & Zoonotic & $n$ & $\times 26$ \\
\hline STM 5229/05 & Chicken & $n$ & $\mathrm{X} 27$ \\
\hline STM 5067/05 & Zoonotic & $\mathrm{n}$ & $\times 27$ \\
\hline STM 6324/03 & Chicken & $n$ & $\times 28$ \\
\hline STM 8322/03 & chicken & $n$ & $x 28$ \\
\hline STM 3079/05 & Cattle & $n$ & $\times 29$ \\
\hline STM 2560/05 & Duck & $n$ & $\times 30$ \\
\hline STM 3865/04 & Chicken & DT104 & $\times 30$ \\
\hline STM 4524/05 & Chicken & $\mathrm{n}$ & $\times 31$ \\
\hline STM 3068/98 & Chicken & DT104 & $\times 32$ \\
\hline STM 1204/05 & Chicken & DT104 & $\times 33$ \\
\hline STM $3077 / 05$ & Swine & $\mathrm{n}$ & X34 \\
\hline STM 5532/04 & Zoonotic & $n$ & $\times 35$ \\
\hline STM 981/04 & Zoonotic & $n$ & $\times 36$ \\
\hline STM 2552/05 & Cattle & $n$ & $\times 37$ \\
\hline STM 3866/05 & Chicken & $n$ & $\times 38$ \\
\hline 17 & Fro & & גo \\
\hline
\end{tabular}

Fig. 3. Dendrogram of cluster analysis of $S$. Typhimurium strains generated by Gel Compar software using the unweighted pair group arithmetic means (UPGMA) methods. $n=$ not determined.

ranging from 11 to 19 with sizes from $20.5 \mathrm{~kb}$ to $1,135 \mathrm{~kb}$. Wide genetic diversity was found among the strains $(\mathrm{F}=$ 0.4 to 1.0). Among these 39 different pulsotypes, 6 were represented by 2 strains each. Three human strains of phage type DT104 isolated in 1970 were indistinguishable
(Profile X1). The rest of the PFGE profiles were unique. Among the 14 human strains, there were 11 PFPs (Fig. 2A), whereas the 33 zoonotic strains had 28 PFPs (Fig. 2B).

Cluster analysis of the pulsotypes of the $47 \mathrm{~S}$. Typhimurium strains based on $70 \%$ similarity generated 2 major clusters, 
I and II (Fig. 3). Cluster I consisted of 10 strains (8 MDR, 1 drug-sensitive human strain, and 1 drug-sensitive zoonotic strain). Cluster II consisted of 21 strains, comprising 16 PFPs divided into 2 subclusters, IIa and IIb. Subcluster IIa consisted of 4 animal strains isolated between 1995 and 2005, each having a unique PFP, 3 MDR, and 1 drugsensitive strains. Subcluster IIb consisted of 17 strains, comprising 12 PFPs. The rest of the 15 PFPs were not included in any distinctive cluster, and each unique profile was represented by 1 strain, except PFP X30, which was represented by 2 strains. The majority of "older" human strains isolated from 1969 to 1977 were grouped in cluster I with similarity $>85 \%$, which suggests that they were very closely related and were distinctly different from those isolated in recent years.

\section{DisCUSSION}

In Malaysia, there is little information on the prevalence of Salmonella Typhimurium. Although the number of Salmonella infections caused by $S$. Typhimurium remains relatively low, it is still important because of increasing isolation of this particular serovar from food and animals in Malaysia (unpublished data).

Phage typing is important for the epidemiology of Salmonella and has been used to describe pandemic clones of $S$. Typhimurium DT104. In Malaysia, there are no phage typing facilities for $S$. Typhimurium, and therefore this is the first report from this region on the differentiation of $S$. Typhimurium by PFGE and PCR. The use of PCR to serotype $S$. Typhimurium has been successfully reported by others [4].

A high level of multidrug resistance was found among the 47 zoonotic and human strains of $S$. Typhimurium, $57.4 \%$ being resistant to 3 or more groups of antimicrobial agents. The most common pattern shared among the strains was ACSSuT. In this study, only 4 S. Typhimurium DT104 had this pattern.

High rates of resistance to tetracycline $(70.2 \%)$ were found among the strains. This finding was lower than those from Korea where $90.0 \%$ of the $S$. Typhimurium animal strains were resistant to tetracycline [33], probably due to this agent being widely used in animal feed as a growth enhancer [9]. High levels of resistance to streptomycin (53\%) and ampicillin (29.7\%) were also found, but these were lower than those from Taiwan, where $82 \%$ and $76 \%$ of 242 strains were resistant to streptomycin and ampicillin, respectively [19].

We found $57 \%$ of the strains were resistant to sulfonamides, being similar to that reported by Lailler et al. [18], where $56 \%$ of $168 S$. Typhimurium strains from cattle, pigs, and poultry were resistant to sulfonamides. In addition, we also observed $27.6 \%$ resistance to nalidixic acid and $19 \%$ to trimethoprim. Numerous studies have reported the increase in resistance of $S$. Typhimurium strains to nalidixic acid and trimethoprim [6, 17], probably as a result of the use of these agents in the treatment of invasive gastrointestinal infections [1] and in animal feeds [30]. Besides the high resistance to nalidixic acid, resistance to ciprofloxacin was found in one human strain isolated in 2006.

Resistance rates of $S$. Typhimurium strains to cephalosporins were higher in the human than in the zoonotic strains, probably due to the use of cephalosporin antibiotics in the treatment of salmonellosis [10].

A multiplex-PCR was applied for accurate detection of antibiotics resistance genes. Our results show that the predominant tetracycline-resistant gene tet $A$ was present in both human and zoonotic strains, followed by $\operatorname{tet} B$ and tet $C$ genes. None of our tetracycline-resistant strains harbored the $t e t G$ gene. However, $\mathrm{Ng}$ et al. [22] found that among $31 \mathrm{~S}$. Typhimurium strains isolated in Canada, tet $G$ was the predominant tetracycline resistance gene present, followed by $t e t B$.

Sulfonamide-resistant gene sul2 was detected among our 27 sulfonamide-resistant strains. The sul2 gene has been found associated with plasmids and not with the class 1 integron [5], but in our study the sul2 gene was found alone in 17 integron-positive strains. The sull gene was detected as part of the class 1 integron in 10 Malaysian strains. Seven strains harbored both sull and sul2 genes, indicating that these strains probably carried class 1 integrons and plasmids.

The aadA gene, which confers resistance to streptomycinspectinomycin, was found as part of the class 1 integron, in agreement with previous studies that it is present in the variable region of class 1 integrons in $S$. Typhimurium $[6,25]$.

The strA gene was the most frequently found in all streptomycin-resistant strains, and has been reported to be associated with plasmids [25]. Both strA and aadA genes were present in 11 strains, which most probably carried plasmids containing the str $A$ gene and the class 1 integron harboring the $\mathrm{aad} A$ gene.

Class 1 integrons were present in $33(70.2 \%)$ strains, indicating a wide distribution of this mobile genetic element among the Malaysian Salmonella Typhimurium strains. Most of the integron-positive strains carried the 210-bp integron, which appears to be unique for $S$. Typhimurium [12] and is part of the pur $\mathrm{G}$ gene. The 800-bp integron harbored $d f r A 7$. Sequence analysis of the 1,000-bp amplicon of different integron profiles showed the presence of aadA2 genes. The persistence and spread of these genes in different Salmonella serotypes might be associated with the extensive use of streptomycin in food animals. Similar results were reported previously $[6,14]$. The 1,200-bp region carried the pse-1 gene (AF153200). Similar integrons were reported in several previous studies $[6,22]$. According to Guerra et al. [14], the presence of 
integrons with variable regions of $1,000 \mathrm{bp}$ (aadA2) and $1,200 \mathrm{bp}$ (pse-1) is very common among multiple resistant DT104 strains. The 1,900-bp amplicon was found in multidrugresistant strains (resistant to more than 5 antimicrobial agents), and sequencing analysis showed that the 1,900-bp amplicon carried 2 genes; the $d f_{r} A 12$ gene, which conferred resistance to trimethoprim, and the aadA2 gene, which conferred resistance to streptomycin-spectinomycin. Guerra et al. [15] also reported both these genes (aadA2 and $d f r$ A12) in the 1,900-bp class 1 integron in Salmonella enterica serotype [4,5,12:i:-] isolated in Spain. Whether the gene cassettes in the integrons are associated with Salmonella Genomic Island I as commonly reported [8] will be determined in future work.

Salmonellae can harbor plasmids of different molecular sizes, ranging from 1 to $200 \mathrm{~kb}$ [27]. In this study, 22 different plasmid profiles with plasmids ranging from 2.0 to $95 \mathrm{~kb}$ were obtained. Ten small plasmids, 2.0, 2.2, 2.64, $3.1,3.4,3.8,4.3,5.4,10.6$, and $11.3 \mathrm{~kb}$, were identified. Similar small plasmid sizes were reported by Biendo et al. [7] in France and Foley et al. [13] in the U.S.A.

Seventeen (48.5\%) plasmid-positive strains harbored the $90-\mathrm{kb}$ plasmid, whereas one strain had the $95-\mathrm{kb}$ plasmid. This particular sized plasmid has been called the Salmonella serovar-specific plasmid, and was reported in several studies [3, 21].

No plasmid was detected in 11 drug-resistant $S$. Typhimurium strains in this study. However, the gain or loss of plasmids is well established, and therefore the analysis of the plasmid profile is not a definitive typing method. During plasmid extraction, shearing of largeplasmid DNA and coprecipitation with the chromosomal DNA are usually reported as one of the causes of plasmid loss [16]. According to Olsen et al. [24], the absence of plasmid in the resistant strains could be due to plasmid instability, a common phenomenon among salmonellae.

No distinct association was found between the resistant phenotypes and $X b a \mathrm{I}$-pulsotypes by cluster analysis of the 47 S. Typhimurium strains. PFGE did not distinguish the drug sensitive and resistant strains, as they shared similar pulsotypes. The human strains isolated between 1969 and 1977 were grouped in one cluster. All except one (28723/ 77) were DT104, indicating that this phage type was prominent in the $S$. Typhimurium population at that period (1969-1977). These strains were very similar (85\%), and were closely related. We identified 22 unique PFGE patterns among the zoonotic $S$. Typhimurium over a period of 10 years (1995-2005). The high diversity of PFGE subtypes suggests that the infections in different animal hosts were from various sources. The majority of older human strains isolated from 1969 to 1977 were grouped in cluster I with similarity $>85 \%$, which suggests that they were very closely related and were distinctly different from those isolated in recent years.
In conclusion, high rates of resistance were found among the strains indicated by the prevalence of resistance genes. Approximately $70.2 \%$ and $74.4 \%$ of the strains carried class 1 integrons and plasmids, which may explain the dissemination of antimicrobial resistance genes through these elements. The existence of multidrug-resistant $S$. Typhimurium to cephalosporins was also observed. PFGE analysis showed that the strains were very diverse and no genotype seemed to predominate and persist over the years.

\section{Acknowledgments}

This study was supported by an eScience Grant (12-02-032024) from MOSTI, Vote F (P0133/2006C) from University of Malaya, and the National Institute of Infectious Diseases, Japan. We thank the Institute of Medical Research, Veterinary Research Institute, and University Malaya Medical Centre for providing some of the strains for study.

\section{REFERENCES}

1. Aarestrup, F. M., C. Wiuff, K. Molbak, and E. J. Threlfall. 2003. Is it time to change fluoroquinolone breakpoints for Salmonella spp.? Antimicrob. Agents Chemother. 47: 827-829.

2. Aarestrup, F. M., M. Lertworapreecha, M. C. Evans, A. Bangtrakulnonth, T. Chalermchaikit, R. S. Hendriksen, and H. C. Wegener. 2003. Antimicrobial susceptibility and occurrence of resistance genes among Salmonella enterica serovar Weltevreden from different countries. J. Antimicrob. Chemother. 52: 715718 .

3. Adaska, J. M., A. J. Silva, A. C. Berge, and W. M. Sischo. 2006. Genetic and phenotypic variability among Salmonella enterica serovar Typhimurium isolates from California dairy cattle and humans. Appl. Environ. Microbiol. 72: 6632-6637.

4. Alvarez, J., M. Sota, A. B. Vivanco, I. Perales, R. Cisterna, A. Rementeria, and J. Garaizar. 2004. Development of a multiplex PCR technique for detection and epidemiological typing of Salmonella in human clinical samples. J. Clin. Microbiol. 42: 1734-1738.

5. Antunes, P., J. Machado, J. C. Sousa, and L. Peixe. 2005. Dissemination of sulfonamide resistance genes (sull, sul2, and sul3) in Portuguese Salmonella enterica strains and relation with integrons. Antimicrob. Agents Chemother. 49: 836-839.

6. Antunes, P., J. Machado, and L. Peixe. 2006. Characterization of antimicrobial resistance and class 1 and 2 integrons in Salmonella enterica isolates from different sources in Portugal. J. Antimicrob. Chemother. 58: 297-304.

7. Biendo, M., G. Laurans, D. Thomas, B. Canarelli, F. HamdadDaoudi, F. Rousseau, S. Castelain, and F. Eb. 2005. Molecular characterisation and mechanisms of resistance of multidrugresistant human Salmonella enterica serovar Typhimurium isolated in Amiens (France). Int. J. Antimicrob. Agents 26: 219-229.

8. Boyd, D. A., G. A. Peters, L.-K. Ng, and M. R. Mulvey. 2000. Partial characterization of a genomic island associated with the 
multidrug resistance region of Salmonella enterica Typhimurium DT104. FEMS Microbiol. Lett. 189: 285-291.

9. Cardoso, M. O., A. R. Ribeiro, L. R. dos Santos, F. Pilotto, H. L. S. de Moraes, C. T. P. Salle, S. L. S. Rocha, and V. P. do Nascimento. 2006. Antibiotic resistance in Salmonella Enteritidis isolated from broiler carcasses. Braz. J. Microbiol. 37: 368371.

10. Chen, S., S. Zhao, D. G. White, C. M. Schroeder, R. Lu, H. Yang, P. F. McDermott, S. Ayers, and J. Meng. 2004. Characterization of multiple-antimicrobial-resistant Salmonella serovars isolated from retail meats. Appl. Environ. Microbiol. 70: $1-7$.

11. Clinical and Laboratory Standards Institute. 2005. Performance Standards for Antimicrobial Susceptibility Testing Approved Standard. M100-S15. Clinical and Laboratory Standards Institute, Wayne, PA.

12. Daly, M., J. Buckley, E. Power, C. O'Hare, M. Cormican, B. Cryan, P. G. Wall, and S. Fanning. 2000. Molecular characterization of Irish Salmonella enterica serotype Typhimurium: Detection of class I integrons and assessment of genetic relationships by DNA amplification fingerprinting. Appl. Environ. Microbiol. 66: 614-619.

13. Foley, S. L., D. G. White, P. F. McDermott, R. D. Walker, B. Rhodes, P. J. Fedorka-Cray, S. Simjee, and S. Zhao. 2006. Comparison of subtyping methods for differentiating Salmonella enterica serovar typhimurium isolates obtained from food animal sources. J. Clin. Microbiol. 44: 3569-3577.

14. Guerra, B., S. Soto, S. Cal, and M. C. Mendoza. 2000. Antimicrobial resistance and spread of class 1 integrons among Salmonella serotypes. Antimicrob. Agents Chemother. 44: 21662169.

15. Guerra, B., S. M. Soto, J. M. Argüelles, and M. C. Mendoza. 2001. Multidrug resistance is mediated by large plasmids carrying a class 1 integron in the emergent Salmonella enterica serotype [4,5,12:i:-]. Antimicrob. Agents Chemother. 45: 1305-1308.

16. Hansen, J. B. and R. H. Olsen. 1978. Isolation of large bacterial plasmids and characterization of the $\mathrm{P} 2$ incompatibility group plasmids pMG1 and pMG5. J. Bacteriol. 135: 227-238.

17. Heurtin-Le Corre, C., P. Y. Donnio, M. Perrin, M. F. Travert, and J. L. Avril. 1999. Increasing incidence and comparison of nalidixic acid-resistant Salmonella enterica subsp. enterica serotype Typhimurium isolates from humans and animals. J. Clin. Microbiol. 37: 266-269.

18. Lailler, R., F. Grimont, Y. Jones, P. Sanders, and A. Brisabois. 2002. Subtyping of Salmonella Typhimurium by pulsed-field gel electrophoresis and comparisons with phage types and resistance types. Pathol. Biol. 50: 361-368.

19. Lauderdale, T. L., F. M. Aarestrup, P. C. Chen, J. F. Lai, H. Y. Wang, Y. R. Shiau, I. W. Huang, C. L. Hung, and TSAR hospitals. 2006. Multidrug resistance among different serotypes of clinical Salmonella isolates in Taiwan. Diagn. Microbiol. Infect. Dis. 55: 149-155.

20. Lévesque, C., L. Pyché, C. Larose, and P. H. Roy. 1995. PCR mapping of integrons reveals several novel combinations of resistance genes. Antimicrob. Agents Chemother. 39: 185-191.

21. Liebana, E., L. Garcia-Migura, C. Clouting, F. A. Clifton-Hadley, E. Lindsay, E. J. Threlfall, S. W. McDowell, and R. H. Davies.
2002. Multiple genetic typing of Salmonella enterica serotype Typhimurium isolates of different phage types (DT104, U302, DT204b, and DT49) from animals and humans in England, Wales, and Northern Ireland. J. Clin. Microbiol. 40: 4450-4456.

22. Ng, L. K., M. R. Mulvey, I. Martin, G. A. Peters, and W. Johnson. 1999. Genetic characterization of antimicrobial resistance in Canadian isolates of Salmonella serovar Typhimurium DT104. Antimicrob. Agents Chemother. 43: 3018-3021.

23. Oliver, A., L. M. Weigel, J. K. Rasheed, J. E. McGowan, P. Raney, and F. C. Tenover. 2002. Mechanisms of decreased susceptibility to cefpodoxime in Escherichia coli. Antimicrob. Agents Chemother. 46: 3829-3836.

24. Olsen, J. E., D. J. Brown, D. L. Baggesen, and M. Bisgaard. 1994. Stability of plasmids in five strains of Salmonella maintained in stab culture at different temperatures. J. Appl. Bacteriol. 77: 155-159.

25. Randall, L. P., S. W. Cooles, M. K. Osborn, L. J. Piddock, and M. J. Woodward. 2004. Antibiotic resistance genes, integrons and multiple antibiotic resistance in thirty-five serotypes of Salmonella enterica isolated from humans and animals in the U.K. J. Antimicrob. Chemother. 53: 208-216.

26. Tassios, P. T., M. Gazouli, E. Tzelepi, H. Milch, N. Kozlova, S. Sidorenko, N. J. Legakis, and L. S. Tzouvelekis. 1999. Spread of a Salmonella typhimurium clone resistant to expandedspectrum cephalosporins in three European countries. J. Clin. Microbiol. 37: 3774-3777.

27. Taylor, D. N., I. K. Wachsmuth, Y. H. Shangkuan, E. V. Schmidt, T. J. Barrett, J. S. Shchrader, et al. 1982. Salmonellosis associated with marijuana: A multistate outbreak traced by plasmid fingerprinting. N. Engl. J. Med. 306: 1249-1253.

28. Thong, K. L., K. S. Lai, and Y. L. Goh. 2003. Technical improvement in DNA preparation in pulsed field electrophoresis. Malaysian J. Sci. 22: 55-59.

29. Threlfall, E. J. 2000. Multiresistant Salmonella Typhimurium DT 104: A truly international multiresistant clone. J. Antimicrob. Chemother. 46: 7-10.

30. Threlfall, E. J. 2002. Antimicrobial drug resistance in Salmonella: Problems and perspectives in food- and water-borne infections. FEMS Microbiol. Rev. 26: 141-148.

31. Wain, J., L. T. Diem Nga, C. Kidgell, K. James, S. Fortune, T. Song Diep, et al. 2003. Molecular analysis of incHI1 antimicrobial resistance plasmids from Salmonella serovar Typhi strains associated with typhoid fever. Antimicrob. Agents Chemother. 47: 2732-2739.

32. Woo, Y. K. and S. H. Lee. 2006. Genetic diversity of multiresistant Salmonella enterica serotype Typhimurium isolates from animals and humans. J. Microbiol. 44: 106-112.

33. Yang, S. J., K.Y. Park, S. H. Kim, K. M. No, T. E. Besser, H. S. Yoo, S. H. Kim, B. K. Lee, and Y. H. Park. 2002. Antimicrobial resistance in Salmonella enterica serovars Enteritidis and Typhimurium isolated from animals in Korea: Comparison of phenotypic and genotypic resistance characterization. Vet. Microbiol. 86: $295-301$.

34. Yasin, R. M., M. M. Jegathesan, and C. C. Tiew. 1997. Salmonella serotypes isolated in Malaysia over the ten-year period 1983-1992. Asia Pac. J. Public Health 9: 1-5. 